\title{
Some cubic birth and death processes and their related orthogonal polynomials
}

\author{
Jacek GILEWICZ* ${ }^{*}$ Elie LEOPOLD *, \\ Andreas RUFFING ${ }^{\dagger}$, Galliano VALENT ${ }^{\ddagger}$ \\ ${ }^{*}$ CNRS Luminy Case 907 \\ Centre de Physique Théorique \\ F-13288 Marseille Cedex 9, France \\ $\dagger$ Zentrum Mathematik \\ Technische Universität München \\ Boltzmannstraße 3, D-85747 Garching, Germany \\ ¥ Laboratoire de Physique Théorique et des Hautes Energies \\ CNRS, Unité associée URA 280 \\ 2 Place Jussieu, F-75251 Paris Cedex 05, France
}

\begin{abstract}
The orthogonal polynomials with recurrence relation

$$
\left(\lambda_{n}+\mu_{n}-z\right) F_{n}(z)=\mu_{n+1} F_{n+1}(z)+\lambda_{n-1} F_{n-1}(z)
$$

with two kinds of cubic transition rates $\lambda_{n}$ and $\mu_{n}$, corresponding to indeterminate Stieltjes moment problems, are analyzed. We derive generating functions for these two classes of polynomials, which enable us to compute their Nevanlinna matrices. We discuss the asymptotics of the Nevanlinna matrices in the complex plane.
\end{abstract}




\section{Introduction}

The field of indeterminate moment problems applied to birth and death processes has been quite active in the past ten years and many explicit examples have been worked out, see [3] and the many references therein. Restricting ourselves to the case of polynomial transition rates $\lambda_{n}$ and $\mu_{n}$ the results obtained dealt mainly with quartic rates [3],[6]. It is the aim of this article to show that the same underlying ideas that led successfully to the computation of the Nevanlinna matrices for the quartic rates can be applied to some cubic rates, leading to some explicit integral representations for their Nevanlinna matrix elements.

The plan of the article is the following. In Section 2 we will recall some basic relations between birth and death processes and orthogonal polynomial theory. In this article we will be concerned with the two processes:

$$
(P 1): \quad \lambda_{n}=(3 n+3 c+1)^{2}(3 n+3 c+2), \quad \mu_{n}=(3 n+3 c-1)(3 n+3 c)^{2}\left(1-\delta_{n 0}\right),
$$

and

$$
(P 2): \quad \lambda_{n}=(3 n+3 c+1)(3 n+3 c+2)^{2}, \quad \mu_{n}=(3 n+3 c)^{2}(3 n+3 c+1)\left(1-\delta_{n 0}\right),
$$

under the assumption $c>0$. In Section 3 some background material useful in the sequel is gathered. In Section 4 and 5 we obtain some generating functions which will allow, in Section 6 to compute the Nevanlinna matrices for both processes. In Section 7 we analyze the asymptotics, in the complex plane, of the Nevanlinna matrix elements.

\section{Birth and death processes versus orthogonal poly- nomials}

Birth and death processes are special stationary Markov processes whose state space is $\mathbb{N}$, representing for instance some population. We are interested in the time evolution of such a population, described by the transition probabilities $\mathcal{P}_{m, n}(t)$ yielding the probability that the population goes from the state $m$ at time $t=0$ to the state $n$ at time $t>0$. This evolution is supposed to be governed by

$$
\begin{aligned}
& \mathcal{P}_{n, n+1}(t)=\lambda_{n} t+o(t), \\
& \mathcal{P}_{n, n}(t)=1-\left(\lambda_{n}+\mu_{n}\right) t+o(t), \quad t \rightarrow 0 . \\
& \mathcal{P}_{n, n-1}(t)=\mu_{n} t+o(t),
\end{aligned}
$$

For applications the most important problem is to find $\mathcal{P}_{m, n}(t)$ for given rates $\lambda_{n}$ and $\mu_{n}$, with suitable extra constraints to be described later on.

From the previous setting one can prove that the transition probabilities have to be a solution of the forward Kolmogorov equations

$$
\frac{d}{d t} \mathcal{P}_{m, n}=\lambda_{n-1} \mathcal{P}_{m, n-1}+\mu_{n+1} \mathcal{P}_{m, n+1}-\left(\lambda_{n}+\mu_{n}\right) \mathcal{P}_{m, n}
$$

The $\mathcal{P}_{m, n}(t)$ are assumed to be continuous for small time scales with

$$
\lim _{t \rightarrow 0} \mathcal{P}_{m, n}(t)=\delta_{m, n}
$$


A representation theorem for $\mathcal{P}_{m, n}(t)$ was proved by Karlin and MacGregor in [5] which links birth and death processes and orthogonal polynomials theory. Let us define the polynomials $F_{n}(x)$ by the three-terms recurrence relation

$$
\left(\lambda_{n}+\mu_{n}-x\right) F_{n}(x)=\mu_{n+1} F_{n+1}(x)+\lambda_{n-1} F_{n-1}(x), \quad n \geq 1
$$

with the initial conditions

$$
F_{0}(x)=1, \quad F_{1}(x)=\frac{\lambda_{0}+\mu_{0}-x}{\mu_{1}} .
$$

Let us define

$$
\pi_{0}=1, \quad \pi_{n}=\frac{\lambda_{0} \lambda_{1} \cdots \lambda_{n-1}}{\mu_{1} \mu_{2} \cdots \mu_{n}}, \quad n \geq 1 .
$$

If the positivity conditions

$$
\lambda_{n}>0, \quad n \geq 0, \quad \text { and } \quad \mu_{0}=0, \quad \mu_{n}>0, \quad n \geq 1
$$

are fulfilled, then there is a positive measure $\psi$ for which

$$
\mathcal{P}_{m, n}(t, \psi)=\frac{1}{\pi_{m}} \int_{\operatorname{supp}(\psi)} e^{-x t} F_{m}(x) F_{n}(x) d \psi(x) .
$$

Then the initial condition (2) is nothing but the orthogonality relation

$$
\frac{1}{\pi_{m}} \int_{\operatorname{supp}(\psi)} F_{m}(x) F_{n}(x) d \psi(x)=\delta_{m, n} .
$$

Such a measure has well-defined moments

$$
c_{n}=\int_{\operatorname{supp}(\psi)} x^{n} d \psi(x), \quad n=0,1, \ldots
$$

If $\operatorname{supp}(\psi) \subseteq \mathbb{R}$ this is a Hamburger moment problem and if $\operatorname{supp}(\psi) \subseteq[0,+\infty[$ this is a Stieltjes moment problem. In the event that the measure $\psi$ is not unique we speak of indeterminate Hamburger (or indeterminate Stieltjes) moment problems, indet $\mathrm{H}$ or indet $\mathrm{S}$ for short. Stieltjes (see [1]) obtained the necessary and sufficient conditions for a moment problem to be indet $\mathrm{S}$

$$
\sum_{n \geq 0} \pi_{n}<\infty, \quad \sum_{n \geq 1} \frac{1}{\lambda_{n} \pi_{n}}<\infty .
$$

These conditions imply that it is also indet $\mathrm{H}$.

Let us consider now the two processes to be analyzed in this article. We will denote the first one as the process $P 1$, with rates

$$
\lambda_{n}=(3 n+3 c+1)^{2}(3 n+3 c+2), \quad \mu_{n}=(3 n+3 c-1)(3 n+3 c)^{2}\left(1-\delta_{n 0}\right), \quad n \geq 0
$$

and the second one as process $P 2$, with rates

$$
\lambda_{n}=(3 n+3 c+1)(3 n+3 c+2)^{2}, \quad \mu_{n}=(3 n+3 c)^{2}(3 n+3 c+1)\left(1-\delta_{n 0}\right), \quad n \geq 0
$$


Using the notation $(a)_{n}=\Gamma(n+a) / \Gamma(a)$ we have for $P 1$ the large $n$ behaviour

$$
\pi_{n}=\left(\frac{(c+1 / 3)_{n}}{(c+1)_{n}}\right)^{2}=\mathcal{O}\left(n^{-4 / 3}\right), \quad \frac{1}{\pi_{n} \mu_{n}}=\mathcal{O}\left(n^{-5 / 3}\right)
$$

and for $P 2$

$$
\pi_{n}=\frac{(c+1 / 3)_{n}\left((c+2 / 3)_{n}\right)^{2}}{\left((c+1)_{n}\right)^{2}(c+4 / 3)_{n}}=\mathcal{O}\left(n^{-5 / 3}\right), \quad \frac{1}{\pi_{n} \mu_{n}}=\mathcal{O}\left(n^{-4 / 3}\right)
$$

These asymptotic estimates show that the conditions (6) are satisfied and therefore the two processes are indet $\mathrm{S}$ and indet $\mathrm{H}$.

\section{Background material}

In order to describe the Nevanlinna matrix we will need a triplet of elementary functions defined by

$$
\sigma_{l}(u)=\sum_{n \geq 0}(-1)^{n} \frac{u^{3 n+l}}{(3 n+l) !}, \quad l=0,1,2 .
$$

It is easy to check the relations

$$
\begin{array}{lll}
\sigma_{1}^{\prime}=\sigma_{0}, & \sigma_{2}^{\prime}=\sigma_{1}, & \sigma_{0}^{\prime}=-\sigma_{2}, \\
\sigma_{0}(0)=1, & \sigma_{1}(0)=0, & \sigma_{2}(0)=0 .
\end{array}
$$

These functions are called trigonometric functions of order 3 , since they are three linearly independent solutions of the third order differential equation

$$
\sigma_{l}^{\prime \prime \prime}+\sigma_{l}=0, \quad l=0,1,2 .
$$

Their explicit form is

$$
\begin{aligned}
& \sigma_{0}(u)=\frac{1}{3}\left(e^{-u}+e^{j u}+e^{\bar{j} u}\right)=\frac{1}{3}\left(e^{-u}+2 \cos \left(\frac{\sqrt{3}}{2} u\right) e^{u / 2}\right), \\
& \sigma_{1}(u)=\frac{1}{3}\left(-e^{-u}+\bar{j} e^{j u}+j e^{\bar{j} u}\right)=\frac{1}{3}\left(-e^{-u}+2 \cos \left(\frac{\sqrt{3}}{2} u-\frac{\pi}{3}\right) e^{u / 2}\right), \quad j=e^{i \pi / 3} . \\
& \sigma_{2}(u)=\frac{1}{3}\left(e^{-u}-j e^{j u}-\bar{j} e^{\bar{j} u}\right)=\frac{1}{3}\left(e^{-u}-2 \cos \left(\frac{\sqrt{3}}{2} u+\frac{\pi}{3}\right) e^{u / 2}\right),
\end{aligned}
$$

We will need also the following functions

$$
\theta(t)=\int_{0}^{t} \frac{d u}{\left(1-u^{3}\right)^{2 / 3}}, \quad \widehat{\theta}(t)=\theta_{0}-\theta(t), \quad \theta_{0} \equiv \int_{0}^{1} \frac{d u}{\left(1-u^{3}\right)^{2 / 3}}=\frac{\Gamma^{3}(1 / 3)}{2 \pi \sqrt{3}} .
$$

Observing that $\widehat{\theta}(t)$ is continuous, decreasing and concave for $t \in[0,1]$ gives the bounds

$$
1-t \leq \frac{\widehat{\theta}(t)}{\theta_{0}} \leq 1
$$




\section{First generating function}

We will consider, for $c>0$, the slightly more general rates than the ones defined in (7):

$$
\begin{array}{ll}
\lambda_{n}=(3 n+3 c+1)^{2}(3 n+3 c+2), & n \geq 0 \\
\mu_{n}=(3 n+3 c-1)(3 n+3 c)^{2}, & n \geq 1 \quad \mu_{0} \geq 0,
\end{array}
$$

where $\mu_{0}$ is taken as a free parameter, not necessarily equal to $(3 c-1)(3 c)^{2}$.

We will denote by $F_{n}\left(z ; c, \mu_{0}\right)$ the polynomials with recurrence relation (3) and the rates (14). Obviously the polynomials corresponding to process $P 1$ are recovered as the limiting values $F_{n}(z ; c, 0)$.

To get most conveniently a generating function for them, it is useful to define a triplet of functions $d_{3 n+l}(\zeta), l=0,1,2$ for $n=0,1, \ldots$ by the recurrence relation

$$
n \geq 0 \quad\left\{\begin{array}{l}
d_{3 n+1}=-\zeta d_{3 n}+\mu_{n} d_{3 n-2}, \\
d_{3 n+2}=-\zeta d_{3 n+1} \\
d_{3 n+3}=-\zeta d_{3 n+2}+\lambda_{n} d_{3 n}
\end{array}\right.
$$

with the initial values

$$
d_{-2}=\frac{1}{\zeta^{2}}, \quad d_{0}=1,
$$

and the definition $\zeta=z^{1 / 3}$. Note that $d_{3 n}(\zeta)$ are polynomials with respect to $z$.

Let us begin with

Proposition 1 The polynomials $F_{n}$ and $d_{n}$ are related by

$$
F_{n}\left(z ; c, \mu_{0}\right)=(3 c) ! \frac{(c+1 / 3)_{n}}{(c+1)_{n}} \frac{d_{3 n}(\zeta)}{(3 n+3 c) !},
$$

where we use the notation $(\alpha) !=\Gamma(\alpha+1)$ for $\alpha>0$.

\section{Proof :}

Let us define $M_{n}(z)=d_{3 n}(\zeta)$. Using (15c) and (15b) in (15) we have for $n \geq 1$

$$
M_{n+1}=d_{3 n+3}=-\zeta d_{3 n+2}+\lambda_{n} d_{3 n}=\lambda_{n} M_{n}+\zeta^{2} d_{3 n+1}=\left(\lambda_{n}-z\right) M_{n}+\zeta^{2}\left(\zeta d_{3 n}+d_{3 n+1}\right) .
$$

Then we use (15a) and (15b) with the shift $n \rightarrow n-1$ to get

$$
M_{n+1}=\left(\lambda_{n}-z\right) M_{n}+\mu_{n} \zeta^{2} d_{3 n-2}=\left(\lambda_{n}-z\right) M_{n}-\mu_{n} \zeta d_{3 n-1} .
$$

The term involving $d_{3 n-1}$ is disposed of using the (15c) with the shift $n \rightarrow n-1$. One is left with

$$
M_{n+1}=\left(\lambda_{n}-z\right) M_{n}+\mu_{n}\left(d_{3 n}-\lambda_{n-1} d_{3 n-3}\right)=\left(\lambda_{n}+\mu_{n}-z\right) M_{n}-\lambda_{n-1} \mu_{n} M_{n-1}, \quad n \geq 1 .
$$

The boundary conditions are to be computed separately and give

$$
M_{0}=1, \quad M_{1}=d_{3}=\lambda_{0}+\mu_{0}-z .
$$


It is then easy to check that the polynomials $F_{n}$ are related to the $M_{n}$ by

$$
F_{n}\left(z ; c, \mu_{0}\right)=\frac{M_{n}(z)}{\mu_{1} \mu_{2} \cdots \mu_{n}}=\frac{d_{3 n}(\zeta)}{\mu_{1} \mu_{2} \cdots \mu_{n}}, \quad n \geq 0 .
$$

Using Gauss multiplication formula we have

$$
\frac{(3 n+3 c) !}{(3 c) \mid}=3^{3 n}(c+1 / 3)_{n}(c+2 / 3)_{n}(c+1)_{n}=\frac{(c+1 / 3)_{n}}{(c+1)_{n}} \mu_{1} \mu_{2} \cdots \mu_{n},
$$

and this leads to the desired relation (16).

In view of Proposition 1 we need generating functions for $d_{3 n+l}$ which we define for the variable $t$ - in a suitable neighbourhood of the origin - as

$$
G_{l}(\zeta, t)=\sum_{n \geq 0} d_{3 n+l}(\zeta) \frac{t^{3 n+3 c+l}}{(3 n+3 c+l) !}, \quad l=0,1,2 .
$$

Routine computations, using relations (15) give for these generating functions the linear differential system

$$
\begin{aligned}
\left(1-t^{3}\right) D_{t} G_{0}-t^{2} G_{0}+\zeta G_{2} & =\frac{t^{3 c-1}}{(3 c-1) !} \\
\left(1-t^{3}\right) D_{t} G_{1}-2 t^{2} G_{1}+\zeta G_{0} & =\frac{\mu_{0}}{\zeta^{2}} \frac{t^{3 c}}{(3 c) !}, \\
D_{t} G_{2}+\zeta G_{1} & =0 .
\end{aligned}
$$

All the factorials involved are well defined in view of the hypothesis $c>0$.

Switching from the functions $G_{i}$ to new functions $\widehat{G}_{i}$ defined by

$$
G_{0}=\frac{\widehat{G}_{0}}{\left(1-t^{3}\right)^{1 / 3}}, \quad G_{1}=\frac{\widehat{G}_{1}}{\left(1-t^{3}\right)^{2 / 3}}, \quad G_{2}=\widehat{G}_{2},
$$

the differential system takes the more symmetric form

$$
\begin{aligned}
\left(1-t^{3}\right)^{2 / 3} D_{t} \widehat{G}_{0}+\zeta \widehat{G}_{2} & =\frac{t^{3 c-1}}{(3 c-1) !} \\
\left(1-t^{3}\right)^{2 / 3} D_{t} \widehat{G}_{1}+\zeta \widehat{G}_{0} & =\frac{\mu_{0}}{\zeta^{2}} \frac{t^{3 c}}{(3 c) !}\left(1-t^{3}\right)^{1 / 3}, \\
\left(1-t^{3}\right)^{2 / 3} D_{t} \widehat{G}_{2}+\zeta \widehat{G}_{1} & =0 .
\end{aligned}
$$

Using the variable $\theta(t)$ defined in (12) we observe that $\left(1-t^{3}\right)^{2 / 3} D_{t}=D_{\theta}$ so that (21) becomes an inhomogeneous differential system with constant coefficients:

$$
D_{\theta} \widehat{G}_{0}+\zeta \widehat{G}_{2}=a(\theta), \quad D_{\theta} \widehat{G}_{1}+\zeta \widehat{G}_{0}=\frac{b(\theta)}{\zeta^{2}}, \quad D_{\theta} \widehat{G}_{2}+\zeta \widehat{G}_{1}=0 .
$$

This is easily solved for $\widehat{G}_{0}$; one gets

$$
\widehat{G}_{0}(\theta)=\int_{0}^{\theta} \sigma_{0}(\zeta(\theta-v)) a(v) d v+\int_{0}^{\theta} \frac{\sigma_{2}(\zeta(\theta-v))}{\zeta^{2}} b(v) d v
$$


From this result we recover $\widehat{G}_{0}(z, t)$ by coming back to the original variable $t$ and after the change of variable $v=\theta^{-1}(u)$ in the integral. Using the notation $\Theta(t, u)=\theta(t)-\theta(u)$, we conclude to:

$$
\begin{aligned}
\widehat{G}_{0}(z, t)=\int_{0}^{t} \sigma_{0}(\zeta \Theta(t, u)) \frac{u^{3 c-1}}{(3 c-1) !}(1 & \left.-u^{3}\right)^{-2 / 3} d u \\
& +\mu_{0} \int_{0}^{t} \frac{\sigma_{2}(\zeta \Theta(t, u))}{\zeta^{2}} \frac{u^{3 c}}{(3 c) !}\left(1-u^{3}\right)^{-1 / 3} d u .
\end{aligned}
$$

Taking into account relations (20), (17) and (16) we have on the one hand

$$
\widehat{G}_{0}(z, t)=\left(1-t^{3}\right)^{1 / 3} G_{0}(z, t)=\frac{1}{(3 c) !} \sum_{n \geq 0} \frac{(c+1)_{n}}{(c+1 / 3)_{n}} F_{n}\left(z ; c, \mu_{0}\right) t^{3 n+3 c}\left(1-t^{3}\right)^{1 / 3}
$$

and on the other hand $\widehat{G}_{0}(z, t)$ given by $(24)$. Gathering all these pieces we end up with Proposition 2 The polynomials $F_{n}\left(z ; c, \mu_{0}\right)$ have the generating function

$$
\begin{aligned}
& \sum_{n \geq 0} \frac{(c+1)_{n}}{(c+1 / 3)_{n}} F_{n}\left(z ; c, \mu_{0}\right) t^{3 n+3 c}\left(1-t^{3}\right)^{1 / 3}= \\
& 3 c \int_{0}^{t} \sigma_{0}(\zeta \Theta(t, u)) u^{3 c-1}\left(1-u^{3}\right)^{-2 / 3} d u+\mu_{0} \int_{0}^{t} \frac{\sigma_{2}(\zeta \Theta(t, u))}{\zeta^{2}} u^{3 c}\left(1-u^{3}\right)^{-1 / 3} d u .
\end{aligned}
$$

This is not quite enough to compute the Nevanlinna matrix; in fact we need

$$
\mathcal{F}\left(z ; c, \mu_{0}\right)=\sum_{n \geq 0} F_{n}\left(z ; c, \mu_{0}\right)
$$

Using the notation

$$
B(\alpha, \beta)=\frac{\Gamma(\alpha) \Gamma(\beta)}{\Gamma(\alpha+\beta)},
$$

we will now state:

Proposition 3 We have the relations

$$
\begin{aligned}
\mathcal{F}\left(z ; c, \mu_{0}\right)=\frac{3}{B(c+1 / 3,2 / 3)}\left\{3 c \int_{0}^{1} u^{3 c-1}\left(1-u^{3}\right)^{-2 / 3} \frac{\sigma_{1}(\zeta \widehat{\theta}(u))}{\zeta} d u\right. & \\
& \left.+\mu_{0} \int_{0}^{1} u^{3 c}\left(1-u^{3}\right)^{-1 / 3} \frac{1-\sigma_{0}(\zeta \widehat{\theta}(u))}{z} d u\right\},
\end{aligned}
$$

valid for $c>0$ and

$$
\begin{gathered}
1-\frac{z}{\mu_{0}} \mathcal{F}\left(z ; c, \mu_{0}\right)=\frac{3}{B(c-2 / 3,2 / 3)} \frac{(3 c-1)(3 c)^{2}}{\mu_{0}} \int_{0}^{1} u^{3 c-3}\left(1-u^{3}\right)^{-1 / 3} \sigma_{0}(\zeta \widehat{\theta}(u)) d u \\
+\frac{\left[\mu_{0}-(3 c-1)(3 c)^{2}\right]}{\mu_{0}} \frac{3}{B(c+1 / 3,2 / 3)} \int_{0}^{1} u^{3 c}\left(1-u^{3}\right)^{-1 / 3} \sigma_{0}(\zeta \widehat{\theta}(u)) d u,
\end{gathered}
$$

valid for $c>1$. 
Proof : In (25) we set $t=\tau^{1 / 3}$, then we multiply both sides by $\tau^{-2 / 3}(1-\tau)^{-2 / 3}$ and integrate from $\tau=0$ to $\tau=1$. The left hand side integral involves a Eulerian integral and we get

$$
\frac{\Gamma(c+1 / 3) \Gamma(2 / 3)}{\Gamma(c+1)} \sum_{n \geq 0} F_{n}\left(z ; c, \mu_{0}\right)=3(3 c) ! \int_{0}^{1} \frac{\widehat{G}_{0}(z, t)}{\left(1-t^{3}\right)^{2 / 3}} d t .
$$

The right hand side is a double integral, which, upon interchange of the order of the integrations and use of relations (10), gives (27). In this last result, the integral with no $\sigma$ function, when expressed in terms of Euler gamma functions simplifies to $\mu_{0} / z$. The first integral in (27), using relations (10), can be integrated by parts twice; then elementary algebra results in (28).

The results obtained so far are sufficient to compute the functions $C_{1}(z)$ and $D_{1}(z)$ in the Nevanlinna matrix of process $P 1$. However, to get the full matrix we need also the generating function for the dual process in the sense of Karlin and McGregor (KMG for short). For the reader's convenience let us recall its definition.

The correspondence from a process $\mathcal{P}$ to its KMG dual $\widetilde{\mathcal{P}}$ is as follows

$$
\mathcal{P}=\left\{\lambda_{n}, \mu_{n}\right\} \quad \rightarrow \quad \widetilde{\mathcal{P}}=\left\{\widetilde{\lambda}_{n}=\mu_{n+1}, \widetilde{\mu}_{n}=\lambda_{n}\right\}
$$

It follows that the dual process of $P 1$ will have

$$
\widetilde{\lambda}_{n}=(3 n+3 c+2)(3 n+3 c+3)^{2}, \quad \widetilde{\mu}_{n}=(3 n+3 c+1)^{2}(3 n+3 c+2), \quad n \geq 0,
$$

which correspond to the process $P 2$ up to the shift $c \rightarrow c+1 / 3$. So we will now work out a generating function for the process $P 2$.

\section{$5 \quad$ Second generating function}

Here again we will consider, for $c>0$, the slightly more general rates than the ones defined in $(8)$ :

$$
\begin{array}{ll}
\lambda_{n}=(3 n+3 c+1)(3 n+3 c+2)^{2}, & n \geq 0 \\
\mu_{n}=(3 n+3 c)^{2}(3 n+3 c+1), & n \geq 1 \quad \mu_{0} \geq 0,
\end{array}
$$

where $\mu_{0}$ is taken as a free parameter, not necessarily equal to $(3 c+1)(3 c)^{2}$.

We will denote by $G_{n}\left(z ; c, \mu_{0}\right)$ the polynomials with recurrence relation $(3)$ and the rates (30). Obviously the polynomials corresponding to process $P 2$ are recovered as $G_{n}(z ; c, 0)$.

In order to avoid repetitions, we will give only the main steps. It is again useful to define a triplet of polynomials $e_{3 n+l}(\zeta), l=0,1,2$ by the recurrence relation

$$
\zeta=z^{1 / 3} \quad n \geq 0 \quad\left\{\begin{array}{l}
e_{3 n+1}=-\zeta e_{3 n}+\mu_{n} e_{3 n-2} \\
e_{3 n+2}=-\zeta e_{3 n+1} \\
e_{3 n+3}=-\zeta e_{3 n+2}+\lambda_{n} e_{3 n}
\end{array}\right.
$$

with the boundary values

$$
e_{-1}=-\frac{1}{\zeta}, \quad e_{0}=1, \quad \Rightarrow \quad e_{3}=\lambda_{0}+\mu_{0}-z
$$

By an argument which follows closely the one given in the proof of Proposition 1, we get: 
Proposition 4 The polynomials $G_{n}$ and $e_{n}$ are related by

$$
G_{n}\left(z ; c, \mu_{0}\right)=(3 c+1) ! \frac{(c+2 / 3)_{n}}{(c+1)_{n}} \frac{e_{3 n}(\zeta)}{(3 n+3 c+1) !} .
$$

We then define the generating functions

$$
H_{l}(\zeta, t)=\sum_{n \geq 0} e_{3 n+l}(\zeta) \frac{t^{3 n+3 c+l}}{(3 n+3 c+l) !}, \quad l=0,1,2
$$

for which we get the differential system

$$
\begin{aligned}
\left(1-t^{3}\right) D_{t} H_{0}-2 t^{2} H_{0}+\zeta H_{2} & =\frac{t^{3 c-1}}{(3 c-1) !} \\
D_{t} H_{1}+\zeta H_{0} & =0 \\
\left(1-t^{3}\right) D_{t} H_{2}-t^{2} H_{2}+\zeta H_{1} & =-\frac{\mu_{0}}{\zeta} \frac{t^{3 c+1}}{(3 c+1) !}
\end{aligned}
$$

Switching from the functions $H_{i}$ to new functions $\widehat{H}_{i}$ defined by

$$
H_{0}=\frac{\widehat{H}_{0}}{\left(1-t^{3}\right)^{2 / 3}}, \quad G_{1}=\widehat{H}_{1}, \quad H_{2}=\frac{\widehat{H}_{2}}{\left(1-t^{3}\right)^{1 / 3}},
$$

and using the variable $\theta(t)$ defined in (12) the previous system becomes an inhomogeneous differential system with constant coefficients, easy to solve. Combining all this we get:

Proposition 5 The polynomials $G_{n}\left(z ; c, \mu_{0}\right)$ have the generating function

$$
\begin{aligned}
& \sum_{n \geq 0} \frac{(c+1)_{n}}{(c+2 / 3)_{n}} G_{n}\left(z ; c, \mu_{0}\right) t^{3 n+3 c+1}=3 c(3 c+1) \int_{0}^{t} \frac{\sigma_{1}(\zeta \Theta(t, u))}{\zeta} u^{3 c-1}\left(1-u^{3}\right)^{-1 / 3} d u \\
&+\mu_{0} \int_{0}^{t} \frac{\sigma_{2}(\zeta \Theta(t, u))}{\zeta^{2}} u^{3 c+1}\left(1-u^{3}\right)^{-2 / 3} d u .
\end{aligned}
$$

In fact we need the generating function

$$
\mathcal{G}\left(z ; c, \mu_{0}\right) \equiv \sum_{n \geq 0} G_{n}\left(z ; c, \mu_{0}\right)
$$

Let us prove:

Proposition 6 We have the relation

$$
\begin{array}{r}
\mathcal{G}\left(z ; c, \mu_{0}\right)=\frac{3}{B(c+2 / 3,1 / 3)}\left\{\begin{array}{r}
3 c(3 c+1) \int_{0}^{1} \frac{\sigma_{2}(\zeta \widehat{\theta}(u))}{\zeta^{2}} u^{3 c-1}\left(1-u^{3}\right)^{-1 / 3} d u \\
+\mu_{0} \int_{0}^{1} \frac{1-\sigma_{0}(\zeta \widehat{\theta}(u))}{z} u^{3 c+1}\left(1-u^{3}\right)^{-2 / 3} d u,
\end{array}\right\}
\end{array}
$$


valid for $c>0$ and

$$
\begin{aligned}
& 1-\frac{z}{\mu_{0}} \mathcal{G}\left(z ; c, \mu_{0}\right)=\frac{3}{B(c-1 / 3,1 / 3)} \frac{(3 c)^{2}(3 c+1)}{\mu_{0}} \int_{0}^{1} u^{3 c-2}\left(1-u^{3}\right)^{-2 / 3} \sigma_{0}(\zeta \widehat{\theta}(u)) d u \\
& +\frac{3}{B(c+2 / 3,1 / 3)} \frac{\mu_{0}-(3 c)^{2}(3 c+1)}{\mu_{0}} \int_{0}^{1} u^{3 c+1}\left(1-u^{3}\right)^{-2 / 3} \sigma_{0}(\zeta \widehat{\theta}(u)) d u
\end{aligned}
$$

valid for $c>1 / 3$.

Proof : In relation (36) we change the variable $t$ to $\tau$ defined by $t=\tau^{1 / 3}$, then multiply both sides by $\tau^{-2 / 3}(1-\tau)^{-2 / 3}$ and integrate from $\tau=0$ to $\tau=1$. The left hand-side is merely a Eulerian integral, while the right-hand side is a double integral. Interchanging the order of integrations, and using relations (10) one gets (38). The integral which does not involve $\sigma$ functions can be expressed in terms of Euler Gamma functions. Then elemetary algebra yields 39 ).

Equipped with these results, let us turn ourselves to the determination of the Nevanlinna matrix for the processes $P 1$ and $P 2$.

\section{The Nevanlinna matrices}

We will write the first Nevanlinna matrix as

$$
\mathcal{N}_{1}(z)=\left(\begin{array}{ll}
A_{1}(z) & C_{1}(z) \\
B_{1}(z) & D_{1}(z)
\end{array}\right) .
$$

As shown in [6] one gets simpler results by considering the modified Nevanlinna matrix

$$
\widetilde{\mathcal{N}}_{1}(z)=\left(\begin{array}{cc}
\widetilde{A}_{1}(z) & C_{1}(z) \\
\widetilde{B}_{1}(z) & D_{1}(z)
\end{array}\right),
$$

where

$$
\widetilde{A}_{1}(z) \equiv A_{1}(z)-\frac{C_{1}(z)}{\alpha}, \quad \widetilde{B}_{1}(z) \equiv B_{1}(z)-\frac{D_{1}(z)}{\alpha}, \quad-\frac{1}{\alpha}=\sum_{n \geq 1} \frac{1}{\mu_{n} \pi_{n}} .
$$

Let us begin with the computation of the modified Nevanlinna matrix for the process $P 1$.

\subsection{The Nevanlinna matrix for P1}

We have first

Proposition 7 The modified Nevanlinna matrix $\widetilde{\mathcal{N}}_{1}(z)$ of process $P 1$ can be expressed in terms of the generating functions $\mathcal{F}$ and $\mathcal{G}$ as

$$
\begin{array}{lll}
\widetilde{A}_{1}(z)=\frac{1}{\lambda_{0}} \mathcal{G}\left(z ; c+1 / 3, \lambda_{0}\right)-\frac{1}{\lambda_{1}} \mathcal{G}\left(z ; c+4 / 3, \lambda_{1}\right), & C_{1}(z)=1-\frac{z}{\mu_{1}} \mathcal{F}\left(z ; c+1, \mu_{1}\right), \\
\widetilde{B}_{1}(z)=-1+\frac{z}{\lambda_{0}} \mathcal{G}\left(z ; c+1 / 3, \lambda_{0}\right), & D_{1}(z)=z \mathcal{F}(z ; c, 0) .
\end{array}
$$




\section{Proof :}

We use successively the relations proved in Lemma 6 of [6]. For the reader's convenience we will recall these relations. We have for the first element

$$
D_{1}(z)=z \sum_{n \geq 0} F_{n}(z ; c, 0)=z \mathcal{F}(z ; c, 0),
$$

upon use of (26). The second element $C_{1}(z)$ is given by

$$
C_{1}(z)=1-\frac{z}{\mu_{0}^{(1)}} \sum_{n \geq 0} F_{n}^{(1)}(z ; c),
$$

where the polynomials $F_{n}^{(1)}(z ; c)$ have the shifted rates

$$
\lambda_{n}^{(1)}=\lambda_{n+1}, \quad \mu_{n}^{(1)}=\mu_{n+1} \quad \Rightarrow \quad F_{n}^{(1)}(z ; c)=F_{n}\left(z ; c+1, \mu_{1}\right) .
$$

Using (26) we conclude to

$$
C_{1}(z)=1-\frac{z}{\mu_{1}} \mathcal{F}\left(z ; c+1, \mu_{1}\right)
$$

The third element is given by

$$
\widetilde{B}_{1}(z)=-1+\frac{z}{\widetilde{\mu}_{0}} \sum_{n \geq 0} \widetilde{F}_{n}(z ; c),
$$

where the $\widetilde{F}_{n}(z ; c)$ are the KMG duals of $F_{n}(z ; c)$, given here by

$$
\widetilde{F}_{n}(z ; c)=G_{n}\left(z ; c+1 / 3, \lambda_{0}\right) \Rightarrow \widetilde{B}_{1}(z)=-1+\frac{z}{\lambda_{0}} \mathcal{G}\left(z ; c+1 / 3, \lambda_{0}\right),
$$

where the last equality follows from (37). The last element is given by

$$
\widetilde{A}_{1}(z)=\frac{1}{\widetilde{\mu}_{0}} \sum_{n \geq 0} \widetilde{F}_{n}(z ; c)-\frac{1}{\widetilde{\mu}_{1}} \sum_{n \geq 0} \widetilde{F}_{n}^{(1)}(z ; c) .
$$

We can write, using (37)

$$
\widetilde{F}_{n}^{(1)}(z ; c)=G_{n}\left(z ; c+4 / 3, \lambda_{1}\right), \quad \widetilde{\mu}_{1}=\lambda_{1},
$$

from which the proposition follows.

The Nevanlinna matrix follows quite easily now:

Proposition 8 The Nevanlinna matrix of the process P1, with rates

$$
\lambda_{n}=(3 n+3 c+1)^{2}(3 n+3 c+2), \quad \mu_{n}=(3 n+3 c-1)(3 n+3 c)^{2}\left(1-\delta_{n 0}\right),
$$

with $c>0$, is given by

$$
\begin{aligned}
& \widetilde{A}_{1}=\frac{3}{B(c+1,1 / 3)} \frac{1}{(3 c+1)} \int_{0}^{1} u^{3 c}\left(1-u^{3}\right)^{-1 / 3} \frac{\sigma_{2}(\zeta \widehat{\theta}(u))}{\zeta^{2}} d u, \\
& \widetilde{B}_{1}=-\frac{3}{B(c+1,1 / 3)} \frac{3 c}{3 c+1} \int_{0}^{1} u^{3 c-1}\left(1-u^{3}\right)^{-2 / 3} \sigma_{0}(\zeta \widehat{\theta}(u)) d u, \\
& C_{1}=\frac{3}{B(c+1 / 3,2 / 3)} \int_{0}^{1} u^{3 c}\left(1-u^{3}\right)^{-1 / 3} \sigma_{0}(\zeta \widehat{\theta}(u)) d u \\
& D_{1}=\frac{3}{B(c+1 / 3,2 / 3)} 3 c z \int_{0}^{1} u^{3 c-1}\left(1-u^{3}\right)^{-2 / 3} \frac{\sigma_{1}(\zeta \widehat{\theta}(u))}{\zeta} d u,
\end{aligned}
$$


where $\zeta=z^{1 / 3}$.

\section{Proof :}

The matrix element $D_{1}$ follows from Proposition 7 and (27). The matrix element $C_{1}$ follows from Proposition 7 and (28). The matrix element $\widetilde{B}_{1}$ follows from Proposition 7 and (28). To compute $\widetilde{A}_{1}$ we use Proposition 7 and (39) to get first

$$
z \widetilde{A}_{1}=-\frac{3}{B(c+1,1 / 3)} \frac{1}{3 c+1} \int_{0}^{1}\left[3 c u^{3 c-1}-(3 c+1) u^{3 c+2}\right]\left(1-u^{3}\right)^{-2 / 3} \sigma_{0}(\zeta \widehat{\theta}(u)) d u .
$$

which is nothing but

$$
z \widetilde{A}_{1}=-\frac{3}{B(c+1,1 / 3)} \frac{1}{3 c+1} \int_{0}^{1} D_{u}\left[u^{3 c}\left(1-u^{3}\right)^{1 / 3}\right] \sigma_{0}(\zeta \widehat{\theta}(u)) d u
$$

An integration by parts gives the required result in Proposition 8 .

Let us observe that the matrix elements $D_{1}$ and $\widetilde{B}_{1}$ can be simplified for $c>1 / 3$. Using relations (10) one realizes that an integration by parts is then possible, leaving us with the matrix elements

$$
\begin{aligned}
& \widetilde{B}_{1}=-\frac{3}{B(c+1,1 / 3)} \frac{1}{3 c+1} \int_{0}^{1} u^{3 c} \frac{\sigma_{1}(\zeta \widehat{\theta}(u))}{\zeta} d u, \\
& D_{1}=\frac{3}{B(c+1 / 3,2 / 3)} \int_{0}^{1} u^{3 c} \zeta \sigma_{2}(\zeta \widehat{\theta}(u)) d u .
\end{aligned}
$$

The special cases where $c=0$ and $c=1 / 3$ lead to considerable simplifications for $B$ and $D$ given in [4].

\subsection{The Nevanlinna matrix for P2}

There is no need to give again the detailed proofs, since everything proceeds as for process $P 1$. Beware that now

$$
\lambda_{n}=(3 n+3 c+1)(3 n+3 c+2)^{2}, \quad \mu_{n}=(3 n+3 c)^{2}(3 n+3 c+1)\left(1-\delta_{n 0}\right) .
$$

We have first

Proposition 9 The modified Nevanlinna matrix $\widetilde{\mathcal{N}}_{2}(z)$ of process P2 can be expressed in terms of the generating functions $\mathcal{F}$ and $\mathcal{G}$ as

$$
\begin{array}{lll}
\widetilde{A}_{2}(z)=\frac{1}{\lambda_{0}} \mathcal{F}\left(z ; c+2 / 3, \lambda_{0}\right)-\frac{1}{\lambda_{1}} \mathcal{F}\left(z ; c+5 / 3, \lambda_{1}\right), & C_{2}(z)=1-\frac{z}{\mu_{1}} \mathcal{G}\left(z ; c+1, \mu_{1}\right), \\
\widetilde{B}_{2}(z)=-1+\frac{z}{\lambda_{0}} \mathcal{F}\left(z ; c+2 / 3, \lambda_{0}\right), & D_{2}(z)=z \mathcal{G}(z ; c, 0) .
\end{array}
$$

Combining this result with the explicit forms of these generating functions, and upon integrations by parts, we get 
Proposition 10 The Nevanlinna matrix of the process P2, with rates

$$
\lambda_{n}=(3 n+3 c+1)(3 n+3 c+2)^{2}, \quad \mu_{n}=(3 n+3 c)^{2}(3 n+3 c+1)\left(1-\delta_{n 0}\right),
$$

where $c>0$, is given by

$$
\begin{aligned}
& \widetilde{A}_{2}=\frac{3}{B(c+1,2 / 3)} \frac{1}{(3 c+2)} \int_{0}^{1} u^{3 c} \frac{\sigma_{2}(\zeta \widehat{\theta}(u))}{\zeta^{2}} d u, \\
& \widetilde{B}_{2}=-\frac{3}{B(c+1,2 / 3)} \frac{3 c}{3 c+2} \int_{0}^{1} u^{3 c-1}\left(1-u^{3}\right)^{-1 / 3} \sigma_{0}(\zeta \widehat{\theta}(u)) d u, \\
& C_{2}=\frac{3}{B(c+2 / 3,1 / 3)}(3 c+1) \int_{0}^{1} u^{3 c} \frac{\sigma_{1}(\zeta \widehat{\theta}(u))}{\zeta} d u, \\
& D_{2}=\frac{3}{B(c+2 / 3,1 / 3)} 3 c(3 c+1) z \int_{0}^{1} u^{3 c-1}\left(1-u^{3}\right)^{-1 / 3} \frac{\sigma_{2}(\zeta \widehat{\theta}(u))}{\zeta^{2}} d u,
\end{aligned}
$$

with $\zeta=z^{1 / 3}$.

Here too, the $c \rightarrow 0$ limit gives again simplifications of the matrix elements $B$ and $D$, see [4].

We will now examine the asymptotics of the entire functions appearing in the Nevanlinna matrix.

\section{Asymptotics of the Nevanlinna matrices}

Three quantities are essential to describe the large $|z|$ behaviour of an entire function $A(z)$, with Taylor series

$$
A(z)=\sum_{n \geq 0} \xi_{n}(A) z^{n}
$$

The order $\rho_{A}$ is defined as

$$
\rho_{A}=\limsup _{n \rightarrow \infty} \frac{n \ln n}{|\ln | \xi_{n}(A) \mid} .
$$

If it is finite, we can define the type $\sigma_{A}$ as

$$
\sigma_{A}=\frac{1}{e \rho_{A}} \limsup _{n \rightarrow \infty} n\left|\xi_{n}(A)\right|^{\rho_{A} / n},
$$

the Phragmén-Lindelöf indicator $h_{A}(\phi)$ being defined by

$$
h_{A}(\phi)=\limsup _{r \rightarrow \infty} \frac{\ln \left|A\left(r e^{i \phi}\right)\right|}{r^{\rho_{A}}}, \quad \phi \in[0,2 \pi] .
$$

The knowledge of the indicator gives the type via the relation

$$
\sigma_{A}=\sup _{\phi \in[0,2 \pi]} h_{A}(\phi)
$$


As a preliminary remark, let us observe that all the matrix elements of the Nevanlinna matrices have the generic structure

$$
N_{l}(z)=\int_{0}^{1} f(u) E_{l}(z, u) d u, \quad f(u)=u^{a}\left(1-u^{3}\right)^{b}, \quad l=0,1,2,
$$

possibly up to a single factor of $z$, appearing in $D_{1}$ and $D_{2}$. We will not care about this factor since it does not change the order, type and Phragmén-Lindelöf indicator.

The entire functions $E_{l}$ which appear have the structure

$$
E_{l}(z, u)=\frac{\sigma_{l}(\zeta \widehat{\theta}(u))}{\zeta^{l}}=\sum_{n \geq 0} \frac{(-1)^{n}}{(3 n+l) !} \widehat{\theta}^{3 n+l}(u) z^{n} .
$$

By inspection, we see that the possible values of the parameters are

$$
a=3 c, 3 c-1, \quad b=0,-1 / 3,-2 / 3
$$

so that in any case we have

$$
a>-1 \text { and } b \geq-2 / 3
$$

and these conditions ensure that $f$ is integrable over $[0,1]$.

Let us begin with

Proposition 11 The order of the entire functions $N_{l}(z), l=0,1,2$ is $\rho=1 / 3$.

Proof :

Since $E_{l}(z, u)$ are entire functions of $z$, uniformly in $u \in[0,1]$, we can integrate term by term in relation (59). This gives

$$
N_{l}(z)=\sum_{n \geq 0} \xi_{l, n} z^{n}, \quad \xi_{l, n}=\frac{(-1)^{n}}{(3 n+l) !} \int_{0}^{1} f(u) \widehat{\theta}^{3 n+l}(u) d u .
$$

From (61) we have

$$
-\frac{\ln \left|\xi_{l, n}\right|}{n \ln n}=\frac{\ln (3 n+l) !}{n \ln n}-\frac{\ln I_{l, n}}{n \ln n}, \quad I_{l, n}=\int_{0}^{1} f(u) \widehat{\theta}^{3 n+l}(u) d u .
$$

In the large $n$ limit, using Stirling formula, this relation yields

$$
\frac{1}{\rho}=3-\limsup _{n \rightarrow \infty} \frac{\ln I_{l, n}}{n \ln n} .
$$

To prove that the second term vanishes in that limit, (because of the logarithm) we need an upper and a lower bound for the integral. Using the inequalities given in (13) we get

$$
\theta_{0}^{3 n+l} \int_{0}^{1} u^{a}(1-u)^{b+l+3 n} d u \leq I_{l, n} \leq \theta_{0}^{3 n+l} M, \quad M=\int_{0}^{1} f(u) d u .
$$

It follows that the logarithm of the upper and lower bounds behave, for large $n$, respectively as

$$
(3 n+l) \ln \theta_{0}-(a+1) \ln n, \quad(3 n+l) \ln \theta_{0}+\ln M
$$


and these imply

$$
\limsup _{n \rightarrow \infty} \frac{\ln I_{l, n}}{n \ln n}=0
$$

and $1 / \rho=3$.

It follows that all the matrix elements of the two Nevanlinna matrices have the same order $1 / 3$. Let us state this result as:

Proposition 12 All the matrix elements of the Nevanlinna matrices given by Proposition 8 and Proposition 10 have one and the same order $\rho=1 / 3$.

Let us now determine the Phragmén-Lindelöf indicator. We first need to prove the following lemma

Lemma 1 Under the hypotheses (60) we have, for $t \rightarrow+\infty$, the asymptotic behaviour

$$
I(t) \equiv \int_{0}^{1} u^{a}\left(1-u^{3}\right)^{b} e^{-t z \theta(u)} d u=\frac{\Gamma(a+1)}{t^{a+1}} e^{-i(a+1) \phi}+\mathcal{O}\left(\frac{1}{t^{a+4}}\right), \quad z=e^{i \phi},
$$

provided that $\phi \in]-\pi / 2,+\pi / 2[$.

\section{Proof :}

The correspondence $u \rightarrow x=\theta(u)$ is a continuous bijection from $[0,1] \rightarrow\left[0, \theta_{0}\right]$. It follows that its inverse function $\theta^{-1}$ is also a continuous bijection. Taking $x$ as a new variable, the integral $I(t)$ can be written as

$$
I(t)=\int_{0}^{\theta_{0}} \chi(x) e^{-t z x} d x, \quad \chi(x)=\left[\theta^{-1}(x)\right]^{a}\left[1-\left(\theta^{-1}(x)\right)^{3}\right]^{b+2 / 3} .
$$

In view of the hypotheses (60) the parameter $b+2 / 3$ is positive and $\chi(x)$ is continuous over $\left.] 0, \theta_{0}\right]$. Let us split the integral into two pieces:

$$
I(t)=I_{1}(t)+I_{2}(t), \quad I_{1}(t)=\int_{0}^{\epsilon} \chi(x) e^{-t z x} d x, \quad I_{2}(t)=\int_{\epsilon}^{\theta_{0}} \chi(x) e^{-t z x} d x
$$

Since $\chi$ is continuous over $\left[\epsilon, \theta_{0}\right]$, we have

$$
\left|I_{2}(t)\right| \leq \sup _{x \in\left[\epsilon, \theta_{0}\right]} \chi(x) \frac{e^{-t(\operatorname{Re} z) \epsilon}-e^{-t(\operatorname{Re} z) \theta_{0}}}{t(\operatorname{Re} z)}
$$

and these terms are vanishing exponentially for large $t$, so they will be negligible when compared to inverse powers of $t$.

Next let us consider $I_{1}$. For sufficiently small $\epsilon$ we can write $\chi(x)=x^{a}+\mathcal{O}\left(x^{a+3}\right)$. So we get

$$
I_{1}(t)=\int_{0}^{\epsilon}\left[x^{a}+\mathcal{O}\left(x^{a+3}\right)\right] e^{-t z x} d x=\int_{0}^{\infty} \cdots-\int_{\epsilon}^{\infty} \cdots .
$$

The second integral in (65) is again exponentially small for large $t$ since we have

$$
\left|\int_{\epsilon}^{\infty} x^{a} e^{-t z x} d x\right| \leq \int_{\epsilon}^{\infty} x^{a} e^{-t(\operatorname{Re} z) x} d x \leq e^{-\epsilon t(\operatorname{Re} z) / 2} \int_{\epsilon}^{\infty} x^{a} e^{-t(\operatorname{Re} z) x / 2} d x
$$


and similarly for the term containing $\mathcal{O}\left(x^{a+3}\right)$. The further bounds

$$
\left|\int_{\epsilon}^{\infty} x^{a} e^{-t z x} d x\right| \leq e^{-\epsilon t(\operatorname{Re} z) / 2} \int_{0}^{\infty} x^{a} e^{-t(\operatorname{Re} z) x / 2} d x=\frac{\Gamma(a+1)}{[t(\operatorname{Re} z) / 2]^{a+1}} e^{-\epsilon t(\operatorname{Re} z) / 2},
$$

show that this term is also with an exponential decrease. The first integral in (65) is well known to give

$$
\left.\int_{0}^{\infty} x^{a} e^{-t z x} d x=\frac{\Gamma(a+1)}{t^{a+1}} e^{-i(a+1) \phi}, \quad z=e^{i \phi}, \quad \phi \in\right]-\frac{\pi}{2},+\frac{\pi}{2}[.
$$

So we end up with ${ }^{1}$

$$
I_{1}(t)=\frac{\Gamma(a+1)}{t^{a+1}} e^{-i(a+1) \phi}+\int_{0}^{\epsilon} \mathcal{O}\left(x^{a+3}\right) e^{-t z x} d x .
$$

For the last piece its modulus is bounded by

$$
\int_{0}^{\epsilon} x^{a+3} e^{-t(\operatorname{Re} z) x} d x \leq \int_{0}^{\infty} x^{a+3} e^{-t(\operatorname{Re} z) x} d x=\mathcal{O}\left(\frac{1}{t^{a+4}}\right)
$$

so the lemma is proved.

In order to get the indicator let us point out that in the Nevanlinna matrix elements, only the entire functions $\sigma_{l}(\zeta \widehat{\theta}(u)) / \zeta^{l}, l=0,1,2$ do appear. Since the factor $\zeta^{l}$ does not change the indicator (see the definition (56)), it is sufficient to deal with the $\sigma_{l}$ and from

relation (11) these can be written as linear combinations of exponentials. Considering a generic matrix element of the form

$$
N(z)=\int_{0}^{1} f(u)\left[a_{0} e^{-\zeta \widehat{\theta}(u)}+a_{+} e^{j \zeta \widehat{\theta}(u)}+a_{-} e^{\bar{j} \zeta \widehat{\theta}(u)}\right], \quad \zeta=r^{1 / 3} e^{i \phi / 3}, \quad z=\zeta^{3},
$$

we will prove:

Proposition 13 All the functions $N$ have as Phragmén-Lindelöf indicator

$$
h(\phi)=\theta_{0} \cos \left(\frac{\phi-\pi}{3}\right), \quad \phi \in[0,2 \pi] .
$$

\section{Proof :}

Let us use the notations

$$
z_{0} \equiv e^{i \phi / 3}=c_{0}+i s_{0}, \quad z_{+} \equiv e^{i(\phi+\pi) / 3}=c_{+}+i s_{+}, \quad z_{-} \equiv e^{i(\phi-\pi) / 3}=c_{-}+i s_{-},
$$

let us determine the Phragmén-Lindelöf indicator first for $\phi \in] 0,2 \pi[$.

It is elementary to check that the following inequalities hold:

$$
\begin{aligned}
& \left.\phi \in] 0, \frac{\pi}{2}\right] \quad c_{-}>c_{+}>0 \quad-c_{0}<0 \\
& \phi \in] \frac{\pi}{2}, \frac{3 \pi}{2}\left[\quad c_{-}>\sqrt{3} / 2 \quad-c_{0}<0, \quad c_{+}<0\right. \\
& \phi \in\left[\frac{3 \pi}{2}, 2 \pi\left[\quad c_{-}>-c_{0}>0 \quad c_{+}<0\right.\right.
\end{aligned}
$$

\footnotetext{
${ }^{1}$ All the equalities are understood up to exponentially small terms omitted.
} 
The asymptotic behaviour of the integral must be analyzed separately for these three cases.

Let us begin with the simplest case, when $\phi \in] \frac{\pi}{2}, \frac{3 \pi}{2}[$. One has

$$
N(z)=e^{z_{-} r^{1 / 3} \theta_{0}} J(r, \theta)
$$

with

$$
J(r, \theta)=a_{-} \int_{0}^{1} f(u) e^{-z_{-} r^{1 / 3} \theta(u)} d u+e^{-z_{-} r^{1 / 3} \theta_{0}} \int_{0}^{1} f(u)\left[a_{+} e^{z_{+} r^{1 / 3} \widehat{\theta}(u)}+a_{0} e^{-z_{0} r^{1 / 3} \widehat{\theta}(u)}\right] d u .
$$

We have merely used the relation $\widehat{\theta}(u)=\theta_{0}-\theta(u)$ in the piece involving $a_{-}$. Since the real parts of $z_{+}$and $-z_{0}$ are negative, the absolute value of the last two integrals is bounded. Furthermore, since the real part of $z_{-}$is positive, for large $r$ the factor $e^{-z_{-} r^{1 / 3} \theta_{0}}$ has an exponentially decreasing absolute value. So we conclude to

$$
\limsup _{r \rightarrow \infty} \frac{\ln \left|N\left(r e^{i \phi}\right)\right|}{r^{1 / 3}}=c_{-} \theta_{0}+\limsup _{r \rightarrow \infty} \frac{\ln \left|a_{-} \int_{0}^{1} f(u) e^{-z_{-} r^{1 / 3} \theta(u)} d u\right|}{r^{1 / 3}} .
$$

The integral appearing in the numerator has a leading behaviour given by Lemma 1

$$
\left|\int_{0}^{1} f(u) e^{-z_{-} r^{1 / 3} \theta(u)} d u\right| \sim \frac{\Gamma(a+1)}{r^{(a+1) / 3}}, \quad r \rightarrow \infty,
$$

the hypothesis $(\phi-\pi) / 3 \in]-\pi / 2, \pi / 2[$ being indeed satisfied. This shows that the last term in the right hand side of (69) vanishes; therefore we have obtained:

$$
\left.h(\phi)=\limsup _{r \rightarrow \infty} \frac{\left|N\left(r e^{i \phi}\right)\right|}{r^{1 / 3}}=\theta_{0} \cos \left(\frac{\phi-\pi}{3}\right), \quad \phi \in\right] \frac{\pi}{2}, \frac{3 \pi}{2}[.
$$

Let us now examine the region $\phi \in] 0, \frac{\pi}{2}\left[\right.$. This time $-c_{0}<0$ but $c_{+}>0$ remains still bounded by $c_{-}$. So using again the relation $\widehat{\theta}(u)=\theta_{0}-\theta(u)$ in the term involving $a_{-}$and $a_{+}$we can write this time

$$
N(z)=e^{z_{-} r^{1 / 3} \theta_{0}} J(r, \theta)
$$

with

$$
\begin{aligned}
J(r, \theta)=a_{-} \int_{0}^{1} f(u) e^{-z_{-} r^{1 / 3} \theta(u)} d u+ & a_{+} e^{-\left(z_{-}-z_{+}\right) r^{1 / 3} \theta_{0}} \int_{0}^{1} f(u) e^{-z_{+} r^{1 / 3} \theta(u)} d u \\
& +a_{0} e^{-z_{-} r^{1 / 3} \theta_{0}} \int_{0}^{1} f(u) e^{-z_{0} r^{1 / 3} \widehat{\theta}(u)} d u
\end{aligned}
$$

The absolute value of the last two integrals vanishes exponentially for large $r$ so we have again the relations (69), (70) from which we conclude similarly that

$$
\left.\left.h(\phi)=\theta_{0} \cos \left(\frac{\phi-\pi}{3}\right), \quad \phi \in\right] 0, \frac{\pi}{2}\right] .
$$

The analysis for $\phi \in\left[\frac{3 \pi}{2}, 2 \pi[\right.$ is similar to the one of the previous interval, up to an interchange of $-c_{0}$ and $c_{+}$. So we have obtained the Phragmén-Lindelöf indicator for $\phi \in] 0,2 \pi[$. We then extend our result to $[0,2 \pi]$ using its continuity property and this ends the proof.

Proposition 13 proves that all the Nevanlinna matrix elements have the same indicator, which we state as 
Proposition 14 The Nevanlinna matrices given by Propositions 8 and 10 have one and the same Phragmén-Lindelöf indicator

$$
h(\phi)=\theta_{0} \cos \left(\frac{\phi-\pi}{3}\right), \quad \phi \in[0,2 \pi] .
$$

It follows from relation (57) that they have one and the same type $\sigma=\theta_{0}$.

\section{Remarks :}

1. Our results agree with the general theorems proved in [2] according to which, for a given Nevanlinna matrix, all of its four elements have the same order, type and PhragménLindelöf indicator. These quantities are therefore intrinsic to a given matrix. Our Propositions 11 and 13 allow us to deal, in one stroke, with the two different Nevanlinna matrices obtained.

2. It may be interesting to observe that the quantities characterizing the asymptotics in the complex plane of the Nevanlinna matrix are independent of the parameter $c$.

3. It was shown in [4], that for $c=0$, the leading term in the asymptotic behaviour of the N-extremal mass points was given by

$$
x_{n}=\left(\frac{2 \pi n}{\sqrt{3} \theta_{0}}\right)^{3}+o\left(n^{3}\right), \quad n \rightarrow \infty .
$$

Since the order is always $1 / 3$ no matter what $c$ is, the leading cubic dependence on $n$ will remain, but there is still an interesting open issue: will the coefficient in front of $n^{3}$ remain independent of $c$ ?

\section{References}

[1] N. I. Akhiezer, The classical moment problem, Oliver and Boyd, Edinburgh (1965).

[2] C. Berg and H. L. Pedersen, "On the order and type of the entire functions associated with an indeterminate Hamburger moment problem", Arkiv. Math., 32 (1994) $1-11$.

[3] C. Berg and G. Valent, "The Nevanlinna parametrization for some indeterminate Stieltjes moment problems associated with birth and death processes", Methods Appl. Anal., 1 (1994) 169-209.

[4] J. Gilewicz, E. Leopold and G. Valent, "New Nevanlinna matrices for orthogonal polynomials related to cubic birth and death processes", Communication at the 7th International Symposium on Orthogonal Polynomials, Copenhagen (2003), to appear.

[5] S. Karlin and J. L. McGregor, "The differential equations of birth-and-death processes, and the Stieltjes moment problem", Trans. Amer. Math. Soc., 85 (1958) $489-546$.

[6] G. Valent, "Co-recursivity and Karlin-McGregor duality for indeterminate moment problems", Constr. Approx., 12 (1996) 531-553. 